\title{
Immune sensing of Candida albicans requires cooperative recognition of mannans and glucans by lectin and Toll-like receptors
}

\author{
Mihai G. Netea, ${ }^{1,2}$ Neil A.R. Gow, ${ }^{3}$ Carol A. Munro, ${ }^{3}$ Steven Bates, ${ }^{3}$ Claire Collins, ${ }^{3}$ \\ Gerben Ferwerda, ${ }^{1,2}$ Richard P. Hobson, ${ }^{3}$ Gwyneth Bertram, ${ }^{3}$ H. Bleddyn Hughes, ${ }^{3}$ \\ Trees Jansen, ${ }^{1,2}$ Liesbeth Jacobs,, ${ }^{1,2}$ Ed T. Buurman, ${ }^{3}$ Karlijn Gijzen, ${ }^{4}$ David L. Williams, ${ }^{5}$ \\ Ruurd Torensma, ${ }^{4}$ Alistair McKinnon, ${ }^{3}$ Donna M. MacCallum, ${ }^{3}$ Frank C. Odds, ${ }^{3}$ \\ Jos W.M. Van der Meer, ${ }^{1,2}$ Alistair J.P. Brown, ${ }^{3}$ and Bart Jan Kullberg, ${ }^{1,2}$ \\ ${ }^{1}$ Department of Medicine, Radboud University Nijmegen Medical Centre, Nijmegen, The Netherlands. ${ }^{2}$ Nijmegen University \\ Center for Infectious Diseases, Nijmegen, The Netherlands. ${ }^{3}$ School of Medical Sciences, Institute of Medical Sciences, University of Aberdeen, \\ Aberdeen, United Kingdom. ${ }^{4}$ Department of Tumor Immunology, Radboud University Nijmegen Medical Centre, Nijmegen, The Netherlands. \\ ${ }^{5}$ Department of Surgery, East Tennessee State University, Johnson City, Tennessee, USA.
}

\begin{abstract}
The fungal pathogen Candida albicans has a multilayered cell wall composed of an outer layer of proteins glycosylated with $N$ - or $O$-linked mannosyl residues and an inner skeletal layer of $\beta$-glucans and chitin. We demonstrate that cytokine production by human mononuclear cells or murine macrophages was markedly reduced when stimulated by $C$. albicans mutants defective in mannosylation. Recognition of mannosyl residues was mediated by mannose receptor binding to $N$-linked mannosyl residues and by TLR4 binding to $O$-linked mannosyl residues. Residual cytokine production was mediated by recognition of $\beta$-glucan by the dectin- $1 /$ TLR2 receptor complex. $C$. albicans mutants with a cell wall defective in mannosyl residues were less virulent in experimental disseminated candidiasis and elicited reduced cytokine production in vivo. We concluded that recognition of $C$. albicans by monocytes/macrophages is mediated by 3 recognition systems of differing importance, each of which senses specific layers of the C. albicans cell wall.
\end{abstract}

\begin{abstract}
Introduction
Invasive Candida albicans infections are a serious clinical threat in patients who are immunosuppressed or undergo major surgical procedures. Mortality associated with disseminated candidiasis can be as high as $30-40 \%$, despite the availability of new antifungal drugs $(1,2)$. Host defense against systemic candidiasis relies mainly on the ingestion and elimination of C. albicans by cells of the innate immune system, especially neutrophils, monocytes, and macrophages (3-6). After activation of these leukocyte populations by C. albicans, release of proinflammatory cytokines such as TNF- $\alpha$, IL- $1 \beta$, IL- 6 , and IFN- $\gamma$ is the first step in the activation of anticandidal innate immune responses. Proinflammatory cytokines activate neutrophils and macrophages to phagocytose the fungus and to release toxic oxygen and nitrogen radicals, thus eliminating the invading pathogen $(7,8)$. The crucial role of proinflammatory cytokines for the host defense against $C$. albicans has been demonstrated by the increased susceptibility to candidiasis of knockout mice lacking these cytokines $(9,10)$. In contrast, antiinflammatory cytokines such as IL-4 and IL-10 have immunosuppressive effects (11). It is currently believed that the balance between pro- and antiinflammatory cytokine production is decisive in determining whether the host defense system is overwhelmed or able to eliminate the fungal pathogens (12).
\end{abstract}

Nonstandard abbreviations used: $\mathrm{MNC}$, mononuclear cell; $\mathrm{MR}$, mannose receptor; PAMP, pathogen-associated molecular pattern; PRR, pattern-recognition receptor; TEM, transmission electron microscopy.

Conflict of interest: The authors have declared that no conflict of interest exists. Citation for this article: J. Clin. Invest. 116:1642-1650 (2006). doi:10.1172/JCI27114.
Stimulation of proinflammatory cytokine production and the activation of innate immunity depend on accurate recognition of an invading pathogen. The basic strategy for recognizing pathogens by the cells of the innate immunity system consists of nonclonal recognition of conserved structures of microorganisms, called pathogen-associated molecular patterns (PAMPs), which are not present in mammalian cells. Several classes of pattern-recognition receptors (PRRs) recognize the various PAMPs, of which TLRs and C-type lectin receptors are probably the most important (13). We and others have recently identified TLR2 and TLR4 as important PRRs for C. albicans (14-17). The mannose receptor (MR) has also been implicated in the stimulation of cytokine production by C. albicans (18), and we have recently demonstrated that the C-type lectin DC-specific ICAM3-grabbing nonintegrin (DC-SIGN) mediates the uptake of C. albicans by dendritic cells (19).

The outer layer of the cell wall of $C$. albicans is enriched with mannoproteins, which represent $30-40 \%$ of the cell wall dry weight (20). The inner layer is composed of chitin and $\beta 1,3-$ and $\beta 1,6$-glucan. Earlier studies have suggested that mannosylated proteins of C. albicans induce cytokine production (21), and C. albicans mannan reportedly interacts with TLR4 (22). It has been suggested that phospholipomannan of C. albicans is recognized by TLR 2 and induces proinflammatory cytokine production (23), whereas $\beta$-glucans were recognized by a complex of TLR2 and dectin-1 $(24,25)$. However, the relative contribution of these PAMPs and their respective receptors to cytokine stimulation by C. albicans is not known.

Despite the progress in understanding the interaction of some of the fungal PAMPs with leukocyte receptors, there is no integrated view of the mechanisms by which the immune system 

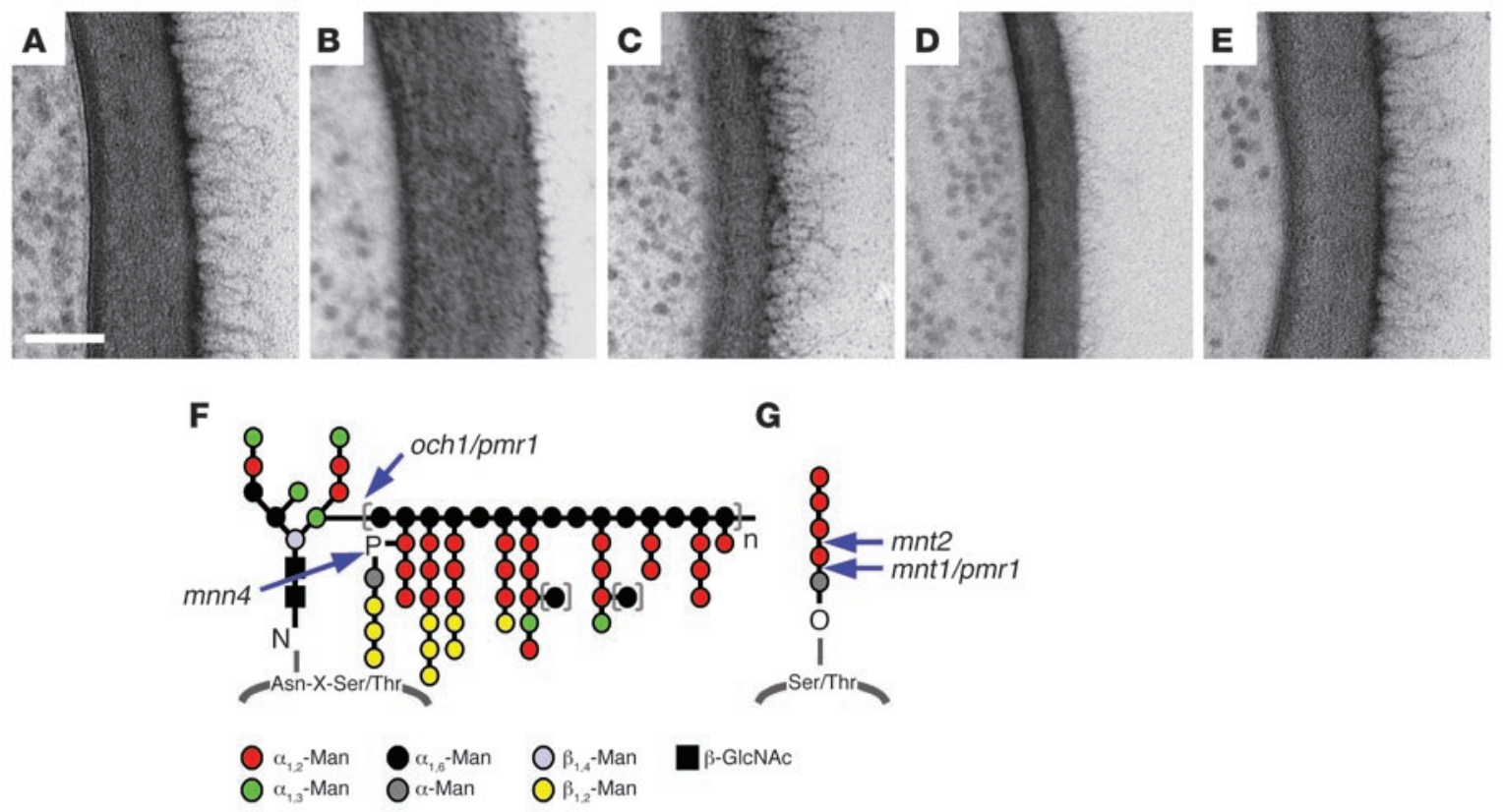

\section{Figure 1}

Cell wall morphology in the C. albicans strains used in this study. (A-E) TEM micrographs. (A) Wild-type strain NGY152 [CAI-4 plus Clp10 vector]. (B) och1 null (strain NGY357; ref. 26) or doxycycline-regulated conditional (strain NGY361; ref. 29) mutants, which are defective in the branched outer $N$-linked mannosyl chains. (C) mnt1 mnt2 mutant (strain NGY337; ref. 27), which lacks 4 terminal O-linked $\alpha_{1,2}$-mannosyl residues. (D) pmr1 mutant (strain NGY355; ref. 26), which has gross defects in mannosylation, characterized by absence of phosphomannan and reduced $O$-linked and $\mathrm{N}$-linked glycans. (E) mnn4 mutant (strain $\mathrm{CDH} 15$; ref. 28), which lacks phosphomannan. Scale bar: $100 \mathrm{~nm}$. (F and $\mathbf{G}) \mathrm{Structure}$ of the $N$ - (F) and $O$-linked (G) glycans and the site of action of deleted gene products. Man, mannosyl; $\beta$-GlcNAc, $\beta N$-acetylglucosamine.

recognizes C. albicans. We hypothesized that recognition of $C$. albicans is a multiple-level process involving specific receptor systems that recognize each layer of the fungal cell wall. The skeleton of the C. albicans cell wall is mainly formed by chitin and $\beta 1,3$ - and $\beta 1,6$-glucans, whereas the outer cell wall is enriched with proteins that are modified with both long-chain and highly branched $N$-linked mannosyl residues as well as short linear chains of $O$-linked mannosyl residues. Using a range of isogenic glycosylation mutants (26-29), ligand-specific blocking experiments, and PAMP receptor knockout mice, we show that recognition of C. albicans by monocytes/macrophages is a complex process involving multiple recognition systems and that these receptor systems recognize sequentially the various layers of the outer portion of the fungal cell wall.

\section{Results}

Cell wall mutants of C. albicans. We have taken a genetic approach to investigate how immune cells recognize a fungal pathogen. We have used well-defined stable isogenic mutants of C. albicans depleted in specific cell wall components to investigate cytokine responses of monocytes. The och1 mutant was defective in outer, branched $N$-linked glycans, and transmission electron microscopy (TEM) revealed a thicker cell wall lacking a fibrillar mannoprotein layer (ref. 29 and compare Figure 1B with Figure 1A). The mnt1 mnt2 mutant lacks the 4 terminal $O$-linked $\alpha_{1,2}$-mannosyl residues, but had normal $N$-mannan (24) and a fibrillar outer wall surface (Figure 1C). The pmr1 mutant has defects in both $\mathrm{N}$ - and $\mathrm{O}$-linked mannosylation, and had a thinner and less fibrillar cell wall than the control strain (Figure 1D). The mnn4 mutant lacks phosphomannan (25) and was indistinguishable by TEM from that of wild-type cells (Figure 1E). Therefore this set of mutants provided a unique molecular biological tool to dissect the immune responses of monocytes.

Mannan stimulates cytokines in a TLR4- and MyD88-dependent manner. Purified mannan (a mixture of both $N$ - and $O$-linked oligosaccharides) from C. albicans stimulated production of TNF and IL-6 in both human mononuclear cells (MNCs) and murine peritoneal macrophages (Figure 2A). The induction of TNF in murine peritoneal macrophages was significantly decreased in mice deficient in MyD88, demonstrating that a TLR-dependent mechanism was involved in this stimulation (Figure 2B). Indeed, the diminished stimulation of macrophages harvested from TLR4-defective ScCr mice demonstrated a TLR4-dependent stimulation of TNF by C. albicans mannan, whereas TLR2-/- mice produced normal amounts of cytokines (Figure 2B). Because purified mannan is a relatively weak cytokine stimulus compared with C. albicans, we investigated whether purified mannan would inhibit C. albicans-induced cytokine production by occupying the mannan-binding sites on leukocytes. The presence of purified mannan significantly inhibited the production of TNF and IL- 6 stimulated by C. albicans, demonstrating that mannan-binding sites are important for the induction of cytokines by the yeast (Figure 2C). In control experiments, mannan had no effect on LPS-induced cytokine production (data not shown). Therefore, fungal mannan stimulated cytokine production, and pretreatment with purified mannan blocked the cytokine response induced by $C$. albicans yeast cells, suggesting an important role of mannan recognition receptors for the stimulation of cytokines by the fungus.

The role of mannosyl residues for cytokine stimulation. The role of global mannosylation of mannoproteins for the stimulation of cytokines by human MNCs was investigated using a pmr1 

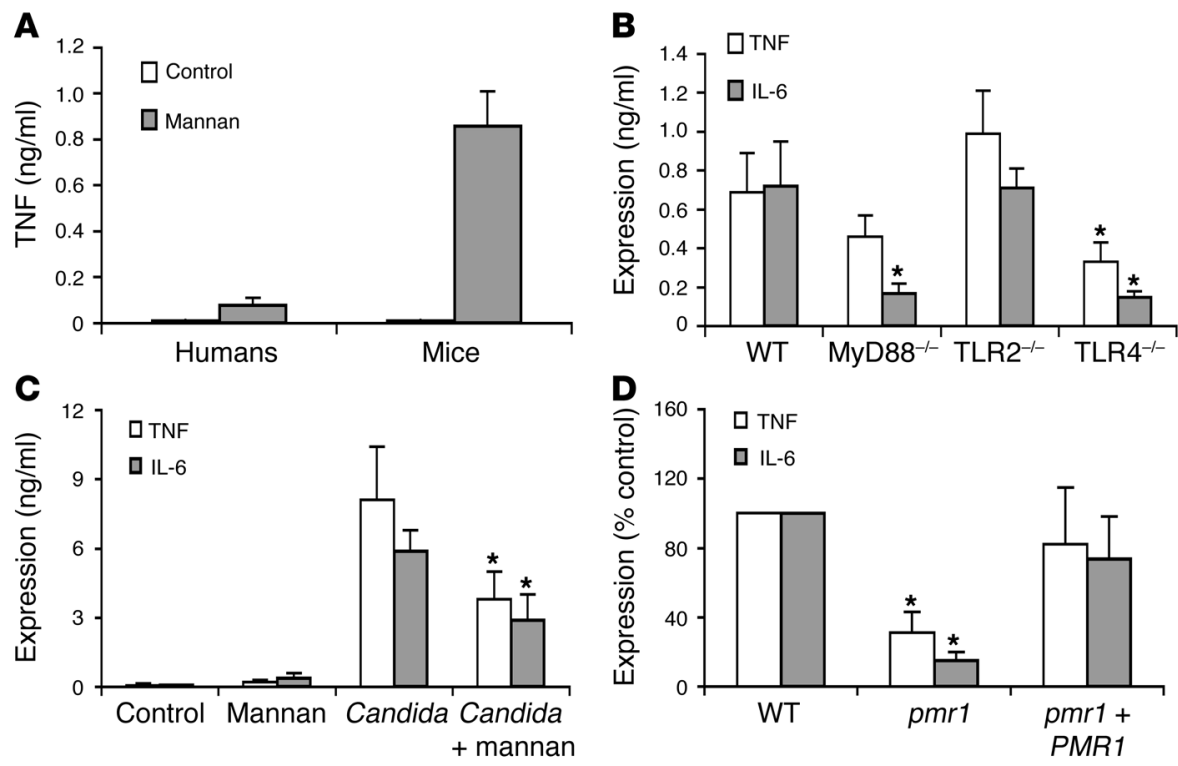

Figure 2

The role of mannan for the cytokine stimulation by C. albicans. (A) Human MNCs or peritoneal macrophages from C57BL/6J mice were stimulated for 24 hours at $37^{\circ} \mathrm{C}$ with $50 \mu \mathrm{g} / \mathrm{ml}$ purified mannan from C. albicans. (B) Peritoneal macrophages from wild-type mice and mice deficient in MyD88, TLR2, or TLR4 were stimulated with mannan. TNF and IL-6 concentrations were measured in the supernatants by specific RIA and ELISA, respectively. Results are pooled data from 2 separate experiments with a total of 10 mice per group. (C) Purified mannan was preincubated for 1 hour at $37^{\circ} \mathrm{C}$ with human MNCs before stimulation with $1 \times 10^{6}$ yeast cells $/ \mathrm{ml}$. Supernatants were collected after additional incubation for 24 hours, and TNF and IL-6 were measured. (D) Human MNCs were stimulated for 24 hours at $37^{\circ} \mathrm{C}$ with whole cells of either wild-type C. albicans (strain NGY152; ref. 26), a mutant defective in the Golgi transporter pmr1 (strain NGY355; ref. 26), or a control strain in which a wild-type copy of $P M R 1$ was introduced into the pmr1 mutant (strain NGY356; ref. 26). TNF and IL-6 concentrations were assessed by RIA and ELISA, respectively. Results (mean $\pm S D$ ) are pooled triplicate data from 2 separate experiments with a total of 8 volunteers per group. ${ }^{*} P<0.05$ versus wild-type.

C. albicans mutant (26). The pmr1 mutant induced significantly less TNF and IL-6 than did the control strain in which PMR1 was reintroduced (Figure 2D). Therefore, normal cytokine stimulation is dependent on mannosylation of the cell wall.

To investigate the specific roles of $N$ - and $O$-linked mannosyl residues for stimulation of cytokines by C. albicans, we compared the stimulation of cytokines by C. albicans strains defective in $N$-linked mannosyl residues (och1), O-linked mannosyl residues (mnt1 mnt2), and mannosylphosphate (mnn4) in human MNCs. The absence of $O$-linked mannosyl residues diminished cytokine release by $30 \%$ in the case of IFN- $\gamma$, whereas the production of TNF was only $15-20 \%$ lower (Figure 3, A and C). In contrast, the absence of $N$-linked mannosyl residues reduced cytokine release by $70 \%$ (Figure 3, A and C). No role of phosphomannan was observed for the induction of cytokine release (Figure 3). Phosphomannan is synthesized preferentially in stationary phase cells; however, no differences were found in cytokine induction by exponential or stationary phase yeast cells (data not shown). An mnn 4 mutant in a serotype B background (30) was also unaffected in its cytokine-inducing properties (data not shown). Similar conclusions were drawn when a time course of the stimulation was performed (Figure 3, C and D). IL-6 release by the various mannosylation mutants displayed a production pattern identical to that of TNF (data not shown).
Normal cytokine release was recovered in strains in which the deleted genes were reintegrated into their respective mutants (Figure 4, A and C). In strain TET-OCH1, the expression of $\mathrm{OCH} 1$ was regulated by placing the $\mathrm{OCH} 1$ open reading frame under the control of a promoter that was regulatable by tetracyclines. Growth in $20 \mu \mathrm{g} / \mathrm{ml}$ doxycycline switched off expression of $\mathrm{OCH} 1$ and resulted in a reduction of cytokine production to a similar level as the och1 mutant. Growth of TET-OCH1 in the absence of doxycycline resulted in expression of $\mathrm{OCH} 1$ and induced cytokine production similar to that of the wildtype strain CAI-4 (Figure 4B).

Similar results were obtained in experiments with live C. albicans cells. The live och1 strain induced only $29 \%$ of the TNF production induced by the control strain $(0.36 \pm 0.11 \mathrm{ng} / \mathrm{ml}$ versus $1.21 \pm 0.23 \mathrm{ng} / \mathrm{ml}$; $P<0.05)$, while the live $m n t 1$ mnt2 strain stimulated $78 \%$ of the control TNF production $(0.94 \pm 0.21 \mathrm{ng} / \mathrm{ml} ; P<0.05)$. In contrast, PBMC stimulated with the $m n n 4$ strain released normal amounts of TNF $(1.79 \pm 0.64 \mathrm{pg} / \mathrm{ml} ; P=\mathrm{NS})$. A similar stimulation pattern was found for other cytokines (data not shown). Using live fungal cells, we assessed the morphology of the fungal elements during the assay $\left(37^{\circ} \mathrm{C}\right.$ incubation in RPMI medium in the absence of plasma or serum). Whereas formation of pseudohyphae was common, no fully developed hyphae were formed. Importantly, there were no differences in hyphal formation among the various mutant strains and the control strain during the incubation.

Recognition of O-linked and $N$-linked mannosyl residues by TLR4 and $M R$. Specific anti-TLR4 and anti-MR blocking antibodies were used to investigate which PRR was involved in the recognition of mannosyl residues of the C. albicans cell wall. Blockade of either TLR4 or MR of human MNCs inhibited TNF production stimulated by the C. albicans wild-type strain NGY152. In contrast, differential blockade of cytokine release was observed in the case of the och1 and mnt 1 mnt 2 mutant strains. The TNF released by the PBMCs stimulated with the $N$-linked mannosylation-defective och1 mutant was inhibited by anti-TLR4, but not by the antiMR antibody (Figure 5A), demonstrating that the MR recognizes $N$-linked mannosyl residues. The opposite was true for TNF stimulation by the $O$-linked mannosylation defective $m n t 1 m n t 2$ mutant, which was inhibited by anti-MR antibodies but not by anti-TLR4 antibodies, suggesting that TLR4 recognizes $O$-linked mannosyl residues (Figure 5A). This conclusion was confirmed in TLR4-deficient mice, which displayed lower TNF production after stimulation with CAI-4 and $o c h 1$ strains but not after stimulation with the mnt 1 mnt2 mutant (Figure 5B).

The role of $\beta$-glucan-dectin-1 interaction for cytokine stimulation. Interaction of $\beta$-glucans with dectin- $1 /$ TLR2 complexes has been shown to induce cytokine production, and DC-SIGN is an additional C-type 
A

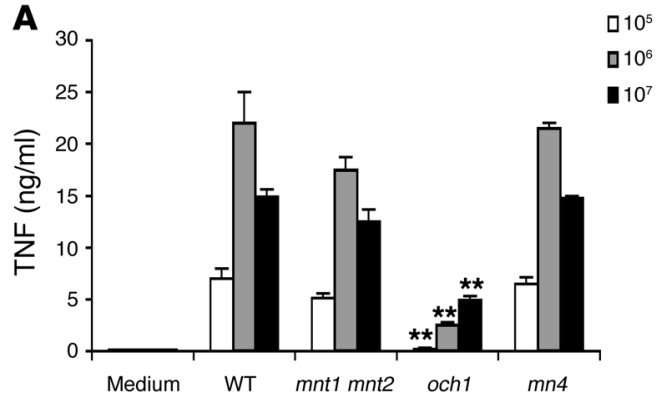

C

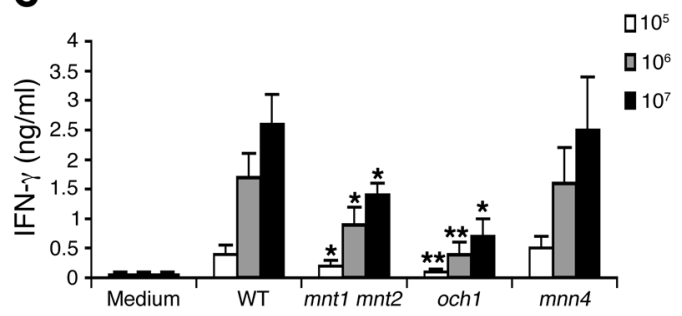

B

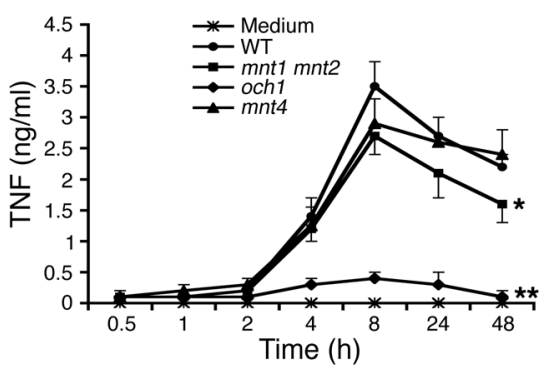

D

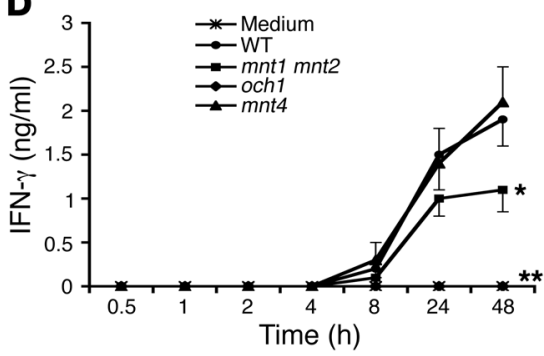

Figure 3

The role of $\mathrm{N}$ - and $\mathrm{O}$-linked mannosyl residues for cytokine stimulation by C. albicans. MNCs were stimulated for various time points with the various C. albicans strains: the wild-type parent NGY152 strain; the och1 mutant (strain NGY357; ref. 29), defective in $\mathrm{N}$-linked mannan; the mnt1 mnt2 mutant (strain NGY337; ref. 27), defective in O-linked mannan; and the mnn4 mutant (strain CDH15; ref. 28), defective in phosphomannan. (A and C) C. albicans concentration-dependent stimulation curves for TNF (A) and IFN- $\gamma(\mathbf{C})$ after stimulation for 24 hours. (B and $\mathbf{D}$ ) Time-dependent stimulation curves for the 2 cytokines when MNCs were stimulated with the various $C$. albicans strains. Results $($ mean $\pm \mathrm{SD})$ are pooled triplicate data from 2 separate experiments with a total of 8 volunteers per group. ${ }^{*} P<0.05$; ${ }^{\star \star} P<0.01$ versus wild-type. lectin receptor that is able to recognize C. albicans. We hypothesized that interaction of $C$. albicans with either of these receptors may account for the residual cytokine production induced in the absence of the mannosyl residues. Accordingly, we used combinations of C. albicans mutant strains and receptor blockade and assessed TNF release by the och1 mutant in TLR $4^{-/-}$mice and by the $m n t 1$ mnt 2 strain in the presence of anti-MR antibodies. In these 2 situations, the signals induced by both $\mathrm{N}$-linked mannosyl/MR and $\mathrm{O}$-linked
mannosyl/TLR4 complexes were absent. The residual cytokine production stimulated by $\mathrm{C}$. albicans in these 2 experimental conditions was completely blocked by laminarin, a ligand of dectin- 1 that is rich in $\beta 1,3$-glucan (Figure 6). In contrast, no role for DC-SIGN could be demonstrated using specific blocking antibodies (data not shown). Interestingly, when dectin-1-blocking experiments were performed with either heat-killed or live C. albicans microorganisms, laminarin had a much stronger inhibitory effect on cytokines

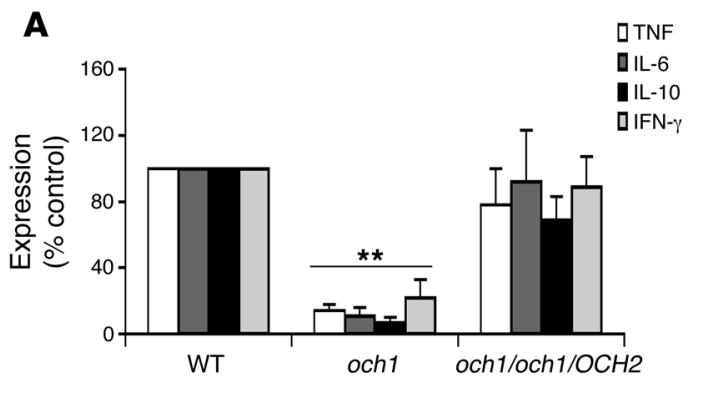

B
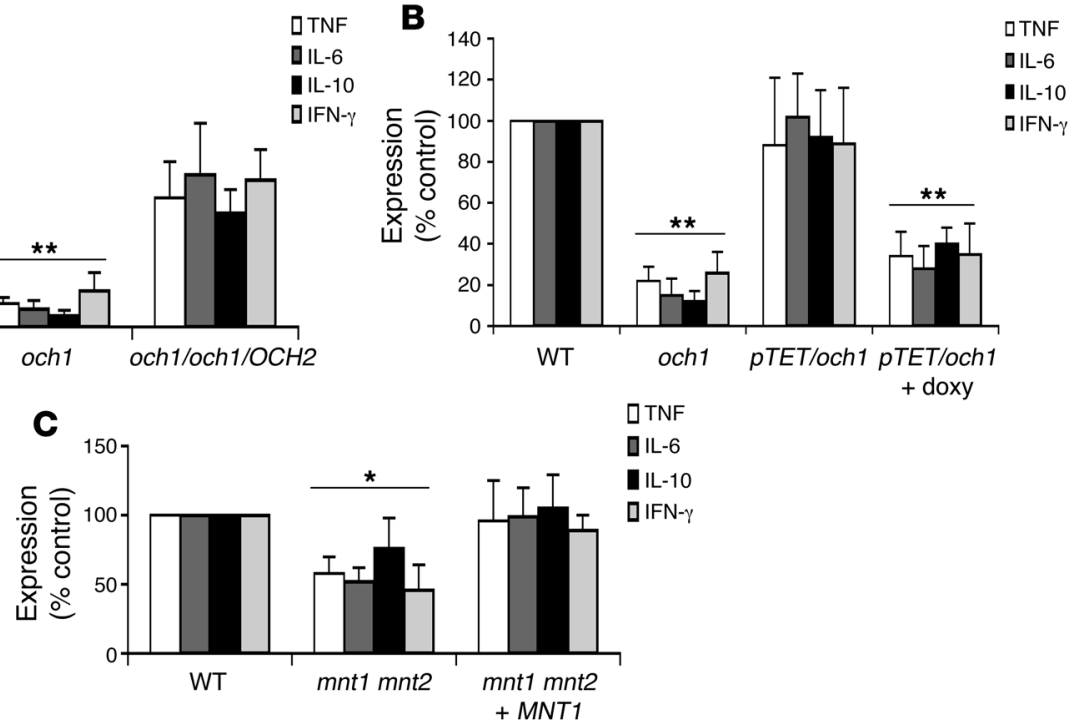

Figure 4

Reintegration of the defective genes restores cytokine production. (A) MNCs were stimulated with the parent NGY152 strain, the $N$-linked mannosyl-defective C. albicans strain (och1; strain NGY357; ref. 29), and the complemented reintegrant och1/och1/OCH1 strain (strain NGY358). (B) Stimulation was also performed with the conditional doxycycline-dependent mutant (pTET/och1; strain NGY361; ref. 29) in both the absence and the presence of doxycycline (doxy). (C) Comparison of the mnt1 mnt2 mutant (strain NGY337; ref. 27), defective in O-linked mannosyl residues, with the mnt1 mnt2 + MNT1 reintegrant strain (strain NGY335; ref. 27). After 24 hours' stimulation at $37^{\circ} \mathrm{C}$, supernatants were collected, and cytokines were determined by RIA or ELISA. Results (mean \pm SD) are pooled triplicate data from 2 separate experiments with a total of 8 volunteers per group. ${ }^{\star} P<0.05 ;{ }^{* \star} P<0.01$ versus wild-type. 

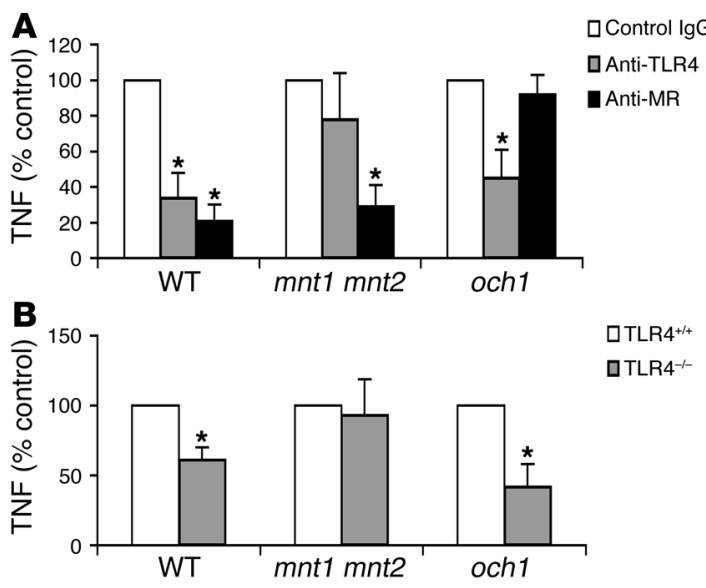

Figure 5

Differential recognition of $\mathrm{O}$ - and $\mathrm{N}$-linked mannosyl residues by TLR4 and MR. (A) Human MNCs were stimulated with the various $C$. albicans strains - the parent NGY152 strain; the och1 mutant (strain NGY357; ref. 29), defective in $\mathrm{N}$-linked mannosylation; and the $\mathrm{mnt} 1 \mathrm{mnt2}$ mutant (strain NGY337; ref. 27), defective in O-linked mannosylation - in the presence of monoclonal antibodies against TLR4 or MR or a isotypematched control antibody. After 24 hours' stimulation at $37^{\circ} \mathrm{C}$, supernatants were collected, and TNF concentration was measured by RIA. Results are pooled triplicate data from 2 separate experiments with a total of 8 volunteers per group. (B) Murine peritoneal macrophages from $\mathrm{TLR}^{+/+} \mathrm{C} 57 \mathrm{BL} / 10 \mathrm{~J}$ and TLR4 ${ }^{-/-} \mathrm{ScCr}$ mice were stimulated with the various C. albicans strains: NGY152, the och1 mutant (NGY357; ref. 29), and the mnt1 mnt2 mutant (NGY337; ref. 27). After 24 hours' stimulation at $37^{\circ} \mathrm{C}$, supernatants were collected, and TNF levels were determined by RIA. Results (mean $\pm S D$ ) are pooled data from 2 separate experiments with a total of 10 mice per group. ${ }^{*} P<0.05$ versus stimulation in the presence of control antibodies $(\mathbf{A})$ or versus TLR $4^{+/+}$mice $(\mathbf{B})$.

induced by heat-killed C. albicans yeasts compared with live fungi (data not shown). This finding strongly sustains the recent findings of Gantner et al., who reported that the $\beta$-glucans of the cell wall in live cells are shielded from recognition by the mannan layers but become exposed in heat-killed C. albicans (31).

The role of mannosyl residues in the virulence of $C$. albicans. Published experiments from our groups have established the role of mannosyl residues in the virulence of C. albicans. We have shown that the och1, pmr1, and $m n t 1$ mnt 2 strains are attenuated in virulence in a mouse model $(26,27)$, but that the mnn4 mutant is unaffected in virulence (28). We extended this in the present study by characterizing the course of disseminated candidiasis and cytokine production in mice

\section{Figure 6}

The role of $\beta$-glucan/dectin-1 interaction for cytokine stimulation by $C$. albicans. The role of $\beta$-glucan/dectin- 1 interaction for $C$. albicansinduced TNF was investigated using 2 approaches with combinations of mutant $C$. albicans strains and receptor blockade: $(\mathbf{A})$ stimulation with the och1 NGY357 strain (29) in TLR4 ${ }^{-/}$mice and (B) stimulation with mnt1 mnt2 NGY337 strain (27) in the presence of anti-MR antibodies in human MNCs. In both situations, the signals induced by $\mathrm{N}$-linked mannosyl/MR and $O$-linked mannosyl/TLR4 complexes were deficient. The residual cytokine production stimulated by $C$. albicans in these 2 experimental conditions was completely blocked by laminarin, a ligand of dectin-1. Results (mean $\pm S D$ ) are pooled data from 2 separate experiments with a total of 10 mice per group $(\mathbf{A})$ or 8 human volunteers (B). ${ }^{*} P<0.05$; ${ }^{* \star} P<0.01$; ${ }^{* \star} P<0.001$ versus wild-type. infected with the och1 C. albicans strain. Compared with the wild-type strain, the och1 strain induced significantly less mortality in a model of disseminated candidiasis in mice (Figure 7A) and had a reduced fungal load in the organs of the mice (Figure 7B). However, viable cells were still present in kidneys with little sign of disease. Virulence was fully recovered in the control strain that contained a reintegrated copy of OCH1 (Figure 7). These virulence parameters were paralleled by significantly decreased cytokine levels in the kidneys of the mice infected with the och1 C. albicans mutant (Figure 7C).

\section{Discussion}

In the present study, we have shown that 3 components of the cell wall of a pathogenic fungus, $N$-linked mannans, $O$-linked mannans, and $\beta$-glucans, are involved in the recognition by monocytes/ macrophages and for the subsequent induction of pro- and antiinflammatory cytokine release. To investigate the role of cell wall mannosyl groups for the recognition of C. albicans, we used isogenic mutant strains of $C$. albicans with specific defects in the mannosylation of cell wall proteins. A gross defect in protein mannosylation was investigated using $C$. albicans $P m r 1 p$, which encodes a $\mathrm{Mn}^{2+} / \mathrm{Ca}^{2+}$ transporter necessary for the activity of several Golgibound mannosyl transferases and shows defects in both $N$-linked and $O$-linked mannosylation (29). The role of phosphomannan was investigated in the mnn4 mutant (28), while the role of $O$-linked mannosylation was assessed using a mnt 1 mnt2 mutant strain lacking 2 partially redundant $\alpha_{1,2}$-mannosyl transferases that are required to add the second and third mannose residues to a linear oligomannoside (27). Finally, the effects of $N$-linked mannosylation were investigated in the och1 C. albicans strain, which lacks an $\alpha_{1,6}$-mannosyl transferase that is required for initiation of the synthesis of the branched outer mannan chains (29).

We demonstrated that $N$-linked and $O$-linked mannosyl groups of glycoproteins of the outer surface of the cell wall were responsible for most of the cytokine-stimulating activity by the yeast
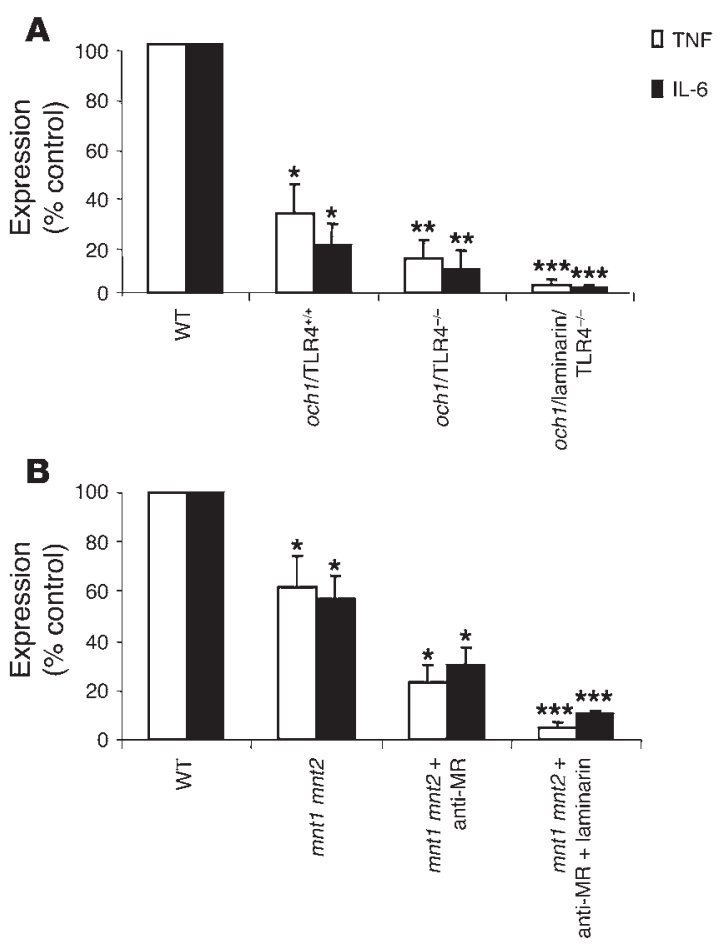
A

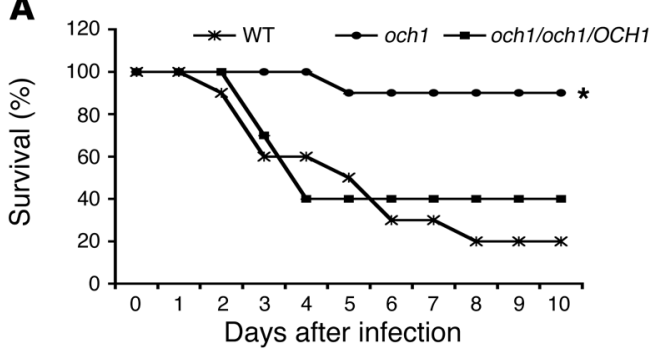

등

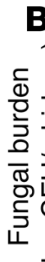

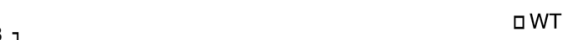
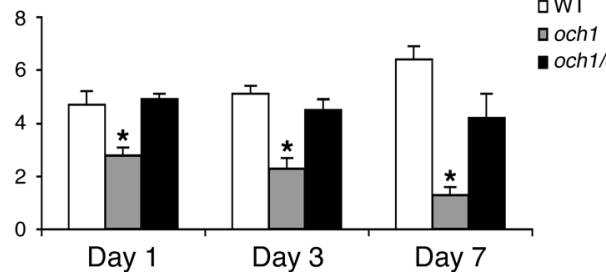
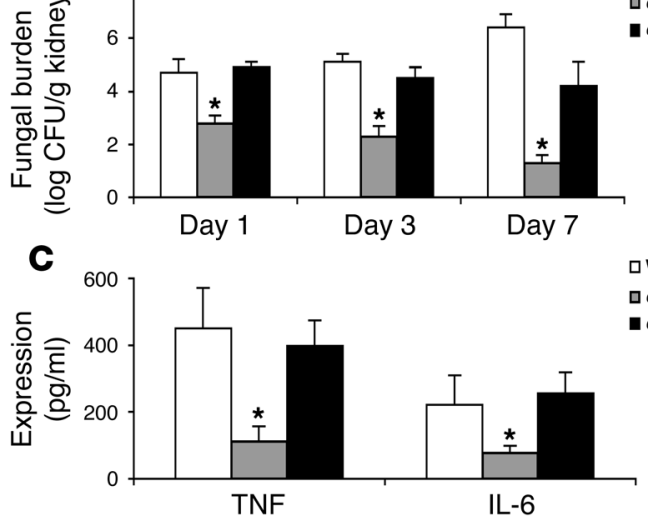

Figure 7

The role of $N$-linked mannosyl residues in virulence and in vivo cytokine production of $C$. albicans. (A) Survival of mice infected intravenously with $5 \times 10^{6} \mathrm{CFU}$ of wild-type C. albicans, the och 1 strain, or the reintegrant control strain. (B) Fungal burden of $C$. albicans in the kidneys of mice infected intravenously with $1 \times 10^{5} \mathrm{CFU}$ of the strains described in A. (C) Cytokine levels on day 3 in the kidneys of mice infected with the strains described in $\mathbf{A}$. Data are presented as means \pm SD. Results are pooled data from 2 separate experiments with a total of 10 mice per group. ${ }^{\star} P<0.05$ versus wild-type.

cell. This was achieved through the specific interaction of the $N$-linked mannosyl residues with MR and of the O-linked mannosyl residues with TLR4. However, the mannosylphosphate fraction of the $N$-linked glycan played practically no role in this process. The residual cytokine production stimulated by C. albicans strains lacking mannosyl residues was mediated by the interaction of $\beta$-glucan with dectin-1, probably in cooperation with TLR2, as discussed below. Thus 3 C. albicans PAMPs cooperate in the activation of the innate immune response, and recognition is a multilevel process involving cooperation between TLRs (TLR4, TLR2) and lectin receptors (MR, dectin-1).

Mannoproteins, $\beta_{1,2}$-oligomannosides, phospholipomannan, and $\beta$-glucans have all been implicated individually in cytokine stimulation induced by C. albicans (21, 32-34). The $\beta 1,3$-glucans are mainly situated in the interior layer of the cell wall of C. albicans, and living yeast cells do not display large amounts of glucans at their surface. $\beta$-glucans become accessible only when the surface mannan coat is removed or damaged by killing, as is the case in zymosan particles that are chemically denuded of mannan (35), or at the site of budding scars in C. albicans yeasts, which are absent from hyphal cells (31). In contrast, $\alpha$ - and $\beta$-linked mannosyl residues are abundant at the surface of the fungus. We found that $\alpha$-linked mannan is critically involved in cytokine stimulation. The $\beta$-linked mannosyl residues are present in the acid-labile and acid-stable fractions of $N$-linked mannans in serotype A strains, in the acid-labile fraction of serotype B strains, and in the structure of phospholipomannan (36). However, cytokine induction was not affected in assays with mnn 4 mutants in either serotype A or serotype B backgrounds (29). The cell wall is therefore a multilayered structure, and it is the external, heavily $\alpha$-linked, mannosylated portion of the cell wall that is most strongly recognized by monocytes.

Mannoproteins have been previously implicated as important cytokine stimuli (21) - our study provides a refined molecular description of this process. The results of our 3 independent approaches support this conclusion. First, we showed that purified mannan isolated from C. albicans stimulated cytokine production in a manner dependent partly on MyD88, an intracellular TLR adaptor molecule (37), and on TLR4. This extends previous studies that also suggested TLR4-dependent cytokine production by mannan (22). Second, we demonstrated that mannan-binding sites on the surface of leukocytes were important for recognition of the yeast cell surface (5). Third, mutants lacking $N$ - or $O$-linked mannosyl residues were markedly affected in their ability to induce cytokines in monocytes.

We demonstrated that a global defect in the mannosylation of cell wall mannoproteins due to in the absence of the pmr 1 Golgi transporter resulted in strongly reduced cytokine induction. Perhaps surprisingly, considering the reported immunostimulatory effects attributed to mannosylphosphate (38), a mannosylphosphate-deficient mnn 4 mutant induced normal cytokine production. This was in agreement with the previous observation that this mutant is recognized normally by macrophages (28). In contrast, approximately $70 \%$ less cytokine production was stimulated by och1 mutants lacking branched $N$-linked mannan, and $30 \%$ less cytokine production was seen using a C. albicans mutant with truncated $O$-linked mannan. A similar difference in cytokine production as found between heat-killed C. albicans strains was seen with the live microorganisms. This is an important observation, because heat-killed C. albicans was present exclusively as yeasts, whereas large numbers of pseudohyphae were present in the preparations using live C. albicans. Therefore, mannans are the most important structure for the induction of cytokines by both live and heat-killed C. albicans, in accord with the localization of these epitopes at the exterior face of the fungal cell wall and with the modifications of the superficial cell wall structure visible by electron microscopy (Figure 1).

In addition to demonstrating the role of protein mannosylation for C. albicans-induced cytokine production, we also identified the PRRs involved in the specific recognition of $N$-linked and $O$-linked mannosyl residues. Using a combination of mannosyl-defective C. albicans strains, anti-receptor blocking antibodies, and knockout mice, we demonstrated that MR recognized the highly branched $N$-linked mannosyl chains whereas TLR4 bound and recognized the linear $O$-linked mannosyl chains. The role of MR $(39,40)$ and TLR4 $(14,22)$ in the recognition of C. albicans has already been proposed, but to our knowledge the C. albicans PAMPs recognized by these receptors have not previously been identified. We have demonstrated previously that differential activation of PRRs such as TLR2 and TLR 4 by C. albicans activates specific responses $(14,15)$, and we and others proposed that this was a universal mechanism of modulation of the innate host defense $(13,41)$. In the present study we showed that the innate immune system was able to 
recognize specific structures of the fungal cell wall, and we are currently investigating how this recognition is translated into differentiated immune responses.

Using combinations of mutant C. albicans strains and receptor blockade (testing the och1 strain in TLR4 ${ }^{-/}$mice and the $m n t 1$ mnt 2 strain in the presence of anti-MR antibodies), we also demonstrated that the residual cytokine production stimulated by $C$. albicans in the absence of signals mediated by mannosyl residues was mediated by the $\beta$-glucan receptor dectin-1. Dectin- 1 has been reported to be the major receptor for $\beta$-glucan (42), and recent studies have demonstrated that it forms a receptor complex with TLR2, amplifying its effects $(24,25)$. Moreover, TLR2-independent activity of dectin-1 has also been recently reported (43). In addition, the recognition of yeasts, but not hyphae, by dectin-1 has been proposed to represent an escape mechanism of the fungus (31).

The differences in pattern recognition and cytokine production among different $C$. albicans strains were reflected by significant differences in the virulence in in vivo models of disseminated candidiasis. In earlier studies we have demonstrated reduced virulence of the pmr 1 , och 1 , and mnt 1 mnt 2 strains $(26,27)$. In the present study we confirmed a significant reduction in the virulence of the $o c h 1$ strain and showed that this strain induced fewer cytokines in vivo (Figure 7). However, the experimental model of candidiasis is more complex than the in vitro experiments. Whereas stimulation of monocytes/macrophages in vitro specifically investigates the role of mannosyl residues for the recognition of C. albicans, the outcome of the experimental infection is influenced by multiple mechanisms, including pattern recognition and cytokine production as well as other factors such as adherence to host endothelial cells and growth rate of the various mutants. The och1 strain not only was defective in cytokine induction, as shown in the present study, but also displays hypersensitivity to agents that perturb the cell wall (29), which may be reflected in a lower resistance to candidacidal mechanisms. Thus a combination of these factors most likely contributes to the lower virulence of the och1 C. albicans mutant.

In conclusion, we show that $C$. albicans induced cytokine stimulation in mammalian MNCs via 3 pathways, each recognizing 1 of the multilayered structures of the fungal cell wall: $N$-linked mannosyl polymers are recognized by MR, $O$-linked chains by TLR4, and $\beta$-glucans by dectin-1/TLR2. Phosphomannan is apparently not involved in pro- or antiinflammatory cytokine induction. The specific activity of the cytokine induction response to these cell wall components reflects their abundance and accessibility in the C. albicans yeast cell wall. This study is the first to our knowledge that describes in totality the recognition pathways of a fungal pathogen and can serve as model for future studies of the innate recognition of other microorganisms.

\section{Methods}

Animals. TLR4-deficient C57BL/ScCr mice were from a local colony at Radboud University Nijmegen, and control TLR4-competent C57BL/10J mice and C57BL/6J mice were obtained from The Jackson Laboratory. MyD88 $/$ mice and TLR2 $/$ - mice on a C57BL/ 6 background were kindly provided by S. Akira (Tokyo University, Tokyo, Japan). All mice weighed 20-25 g and were $6-8$ weeks old. The mice were fed sterilized laboratory chow (Hope Farms) and water ad libitum. The experiments were approved by the ethics committee on animal experiments of Radboud University Nijmegen.

C. albicans strains and growth conditions. Homozygous null mutants in glycosylation genes were constructed in the C. albicans CAI-4 serotype A background by targeted gene disruption (44). Control strains were used in which the wild-type genes, under the control of their own promoters, and the URA3 selectable marker were reintroduced at the neutral RPS1 locus via the CIp10 integrative plasmid (45). Parental strain CAI-4 was also transformed with empty CIp10, generating strain NGY152, making all strains isogenic with regard to Ura status. In addition, a conditional och1 mutant was also used in which 1 remaining functional allele was regulated by the tetracycline/doxycycline-repressable TET promoter (strain NGY361; ref. 29). Mutants had defects in outer chain $N$-mannosylation (och1; strain NGY357; ref. 29), O-mannosylation ( $m n t 1$ mnt2; strain NGY337; ref. 27), and phosphomannan biosynthesis ( $m n n 4$; strain $\mathrm{CDH} 15$; ref. 28) or were downregulated in glycosylation due to low levels of $\mathrm{Mn}^{2+}$ in the Golgi (pmr1; strain NGY355; ref. 26). C. albicans was grown with continuous shaking at $200 \mathrm{rpm}$ at $30^{\circ} \mathrm{C}$ in Sabouraud broth ( $1 \%$ mycological peptone $/ 4 \%$ glucose) overnight, transferred to fresh medium, and incubated for 4 hours. All mutant strains showed similar morphology (yeasts and hyphae) to the control C. albicans strain, either when incubated in growth medium or together with PBMCs, as described previously in detail (26-29). The cells were harvested by centrifugation, and the pellets were washed twice in $20 \mathrm{ml}$ sterile PBS and resuspended to a density of $1 \times 10^{8}$ cells/ml before heat-killing at $56^{\circ} \mathrm{C}$ for 1 hour. The TET-OCH1 strain was pregrown overnight in the presence of $20 \mu \mathrm{g} / \mathrm{ml}$ doxycycline. In separate experiments, live C. albicans yeast cells were washed and resuspended in RPMI 1640 at a concentration of $1 \times 10^{6} \mathrm{CFU} / \mathrm{ml}$ and used for the stimulation of cytokine production. The mnt 1 mnt 2 , $o c h 1$, and pmr1 mutant strains tended to aggregate more easily in culture medium, and we cannot completely exclude a certain degree of aggregation even after disrupting them by vigorous vortexing. This may have resulted in some underestimation of the yeast number in the suspension containing these mutant strains. In contrast, the mnn 4 mutant did not aggregate.

Freeze substitution TEM. Midexponential phase yeast cells were grown in yeast extract, peptone, and dextrose medium and harvested by centrifugation, and the pellets were resuspended in $1 \%$ agarose and transferred to flat specimen carriers. The samples were frozen in liquid nitrogen at high pressure using a Leica EM PACT high-pressure freezer (Leica Microsystems). Freeze substitution of the frozen cells was carried out in an automatic temperature-controlled freeze substitution system (AFS; Leica Microsystems) in dried acetone containing $1 \% \mathrm{OsO}_{4}$ at $-90^{\circ} \mathrm{C}$ for 48 hours. The samples were gradually warmed to $-30^{\circ} \mathrm{C}$ and then processed in a Lynx tissue processor to finish in acetone/resin at a ratio of 1:2. The samples were transferred to a Lynx tissue processor to embed in TAAB812 epoxy resin (TAAB Laboratory Equipment Ltd.). Ultra-thin sections $(60 \mathrm{~nm})$ were cut with a Leica ultracut $\mathrm{E}$, and the sections were stained with uranyl acetate and lead citrate. Samples were imaged in a Philips CM10 transmission microscope (FEI UK Ltd.), and the images were recorded with a Gatan Bioscan 792 (Gatan).

Fungal carbobydrate polymers. The mannan from C. albicans was isolated as previously described by Kogan et al. (46). Briefly, $100 \mathrm{~g}$ wet wt of yeast biomass was suspended in $400 \mathrm{ml} 2 \%$ (wt/vol) $\mathrm{KOH}$ and heated for 1 hour at $100^{\circ} \mathrm{C}$. Insoluble residues were separated by centrifugation, and mannan was precipitated from supernatant with Fehling's reagent. The sedimented mannan-copper complex was dissolved in a minimum volume of $3 \mathrm{M} \mathrm{HCl}$ and added dropwise to methanol/acetic acid at a ratio of $8: 1$ ( $\mathrm{vol} / \mathrm{vol})$. The procedure of dissolution and precipitation was repeated twice. Finally, the sediment was separated, dissolved in distilled water, and dialysed for 24 hours. Mannan contained no nitrogen as determined by elementary analysis. Laminarin was purchased from Sigma-Aldrich. The mannan and laminarin were chemically characterized to confirm their molecular weight and chemical structure $(47,48)$ and were assayed to confirm the absence of endotoxin.

Antibodies. The monoclonal anti-TLR4 HTA125 antibody was a kind gift of K. Miyake (Saga Medical School, Saga, Japan). The monoclonal mouse anti-human MR antibody and the isotype-matched IgG antibody used as a control in all experiments were purchased from Sigma-Aldrich. 
Stimulation of cytokine production in buman MNCs. Isolation of MNCs was performed as described previously (49). Venous blood was collected from cubital veins of 8 healthy volunteers. All volunteers gave informed consent prior to participating in the study. Samples of $5 \times 10^{5}$ MNCs in a $100-\mu \mathrm{l}$ volume were added to round-bottomed 96-well plates (Greiner Bio-One) and incubated for 24 hours with $100 \mu \mathrm{l}$ of the various strains of live or heat-killed $\left(30\right.$ minutes at $\left.56^{\circ} \mathrm{C}\right) \mathrm{C}$. albicans at a concentration $1 \times 10^{6}$ yeast cells $/ \mathrm{ml}$ unless otherwise indicated. In receptor-blocking studies, MNCs were preincubated for 1 hour at $37^{\circ} \mathrm{C}$ with the various monoclonal antibodies (anti-TLR4, anti-MR, or control IgG; $10 \mu \mathrm{g} / \mathrm{ml}$ ) before stimulation with C. albicans. After 24 hours' incubation at $37^{\circ} \mathrm{C}$, the $\mathrm{MNC} / \mathrm{C}$. albicans cell suspensions were centrifuged, and the supernatants were collected and stored at $-70^{\circ} \mathrm{C}$ until assayed. Human TNF- $\alpha$ concentrations were determined by specific RIAs as described previously (50). IL-6, IL-10, and IFN- $\gamma$ concentrations were measured by commercial ELISA kits (Sanquin).

Cytokine production by murine peritoneal macrophages. Resident peritoneal macrophages from the various mouse strains were harvested by injecting $4 \mathrm{ml}$ sterile PBS containing $0.38 \%$ sodium citrate (8). After centrifugation and washing, the cells were resuspended in RPMI 1640. Cells were cultured in 96-well plates and stimulated with $100 \mu \mathrm{l}$ of the various $C$. albicans strains for 24 hours at $37^{\circ} \mathrm{C}(50)$. Murine IL- $1 \alpha$, IL-1 $\beta$, and TNF- $\alpha$ were determined by specific RIAs (detection limit, 20 pg/ml) (51).

C. albicans infection model. We used a C. albicans infection model as described previously (15). Briefly, $1 \times 10^{5}$ (for survival experiments) or $1 \times 10^{5}$ CFUs (for fungal burden) of C. albicans strains (wild-type, och1, or the reintegrant strain) were injected i.v. into mice on day 0 . Survival was assessed daily. On days 3, 7, and 14, kidneys and livers of subgroups of mice were aseptically removed, weighed, and homogenized in sterile saline in a tissue grinder. The number of viable C. albicans cells was determined by plating serial dilutions on Sabouraud dextrose agar plates. The colonies were counted after 24 hours at $37^{\circ} \mathrm{C}$, and results were expressed as CFU/g tissue. In addition, tissue homogenates were centrifuged, and cytokines were measured in the supernatant by ELISA (see above).

Statistics. The human experiments were performed using triplicate samples in 2 experiments with a total of 8 volunteers. The mouse experiments were performed twice in 10 mice per group. The differences between groups were analyzed by Mann-Whitney $U$ test. The level of significance between groups was set at $P<0.05$. Data are given as means $\pm \mathrm{SD}$.

\section{Acknowledgments}

We thank D. Singleton and K. Hazen for the mnn4 serotype B strain. This study was partly supported by a Vidi grant of the Netherlands Organization for Scientific Research to M.G. Netea. N.A.R. Gow, A.J.P. Brown, and F.C. Odds acknowledge financial support from the Wellcome Trust (grants 06324 and 72263). D.L. Williams was supported in part by Public Health Service grants GM53522 from the National Institute of General Medical Sciences and AI45829 from the National Institute of Allergy and Immunology.

Received for publication October 12, 2005, and accepted in revised form April 11, 2006.

Mihai G. Netea and Neil A.R. Gow contributed equally to this work.

Address correspondence to: Mihai G. Netea, Department of Medicine (541), Radboud University Nijmegen Medical Center, PO Box 9101, Geert Grooteplein 8, 6500 HB Nijmegen, The Netherlands. Phone: 31-24-3618819; Fax: 31-24-3541734; E-mail: m.netea@aig.umcn.nl.
1. Pfaller, M.A., et al. 1999. International surveillance of blood stream infections due to Candida species in the European SENTRY Program: species distribution and antifungal susceptibility including the investigational triazole and echinocandin agents. Diagn. Microbiol. Infect. Dis. 35:19-25.

2. Edmond, M.B., et al. 1999. Nosocomial bloodstream infections in United States hospitals: a three-year analysis. Clin. Infect. Dis. 29:239-244.

3. Van 't Wout, J.W., Linde, I., Leijh, P.C.J., and Van Furth, R. 1988. Contribution of granulocytes and monocytes to resistance against experimental disseminated Candida albicans infections. Eur. J. Clin. Microbiol. Infect. Dis. 7:736-741.

4. Kullberg, B.J., Van 't Wout, J.W., and Van Furth, R. 1990. Role of granulocytes in enhanced host resistance to Candida albicans induced by recombinant interleukin-1. Infect. Immun. 58:3319-3324.

5. Marodi, L., Korchak, H.M., and Johnston, R.B., Jr. 1991. Mechanisms of host defense against Candida species. 1. Phagocytosis by monocytes and monocytederived macrophages. J. Immunol. 146:2783-2789.

6. Qian, Q., Jutila, M.A., Van Rooijen, N., and Cutler, J.E. 1994. Elimination of mouse splenic macrophages correlates with increased susceptibility to experimental disseminated candidiasis. J. Immunol. 152:5000-5008.

7. Djeu, J.Y. 1990. Role of tumor necrosis factor and colony-stimulating factors in phagocyte function against Candida albicans. Diagn. Microbiol. Infect. Dis. 13:383-386.

8. Kullberg, B.J., Van 't Wout, J.W., Hoogstraten, C., and Van Furth, R. 1993. Recombinant interferon- $\gamma$ enhances resistance to acute disseminated Candida albicans infection in mice. J. Infect. Dis. 168:436-443.

9. Netea, M.G., et al. 1999. The increased susceptibility of TNF $\alpha$ LT $\alpha$ double knock-out mice to systemic candidiasis is due to defective recruitment and phagocytosis by neutrophils. J. Immunol. 163:1498-1505.
10. Kaposzta, R., Tree, P., Marodi, L., and Gordon, S. 1998. Characteristics of invasive candidiasis in gamma interferon- and interleukin-4-deficient mice: role of macrophages in host defense against Candida albicans. Infect. Immun. 66:1708-1717.

11. Tonnetti, L., et al. 1995. Interleukin-4 and -10 exacerbate candidiasis in mice. Eur. J. Immunol. 25:1559-1565.

12. Romani, L. 2004. Immunity to fungal infections. Nat. Rev. Immunol. 4:1-13.

13. Netea, M.G., Van der Graaf, C., Van der Meer, J.W.M., and Kullberg, B.J. 2004. Toll-like receptors and the host defense against microbial pathogens: bringing specificity to the innate-immune system. J. Leukoc. Biol. 75:749-755

14. Netea, M.G., et al. 2002. The role of toll-like receptor (TLR) 2 and TLR4 in the host defense against disseminated candidiasis. J. Infect. Dis. 185:1483-1489.

15. Netea, M.G., et al. 2004. Toll-like receptor 2 inhibits cellular responses against Candida albicans through pathways mediated by IL-10 and CD4+CD25+ regulatory T cells. J. Immunol. 172:3712-3718.

16. Bellocchio, S., et al. 2004. The contribution of Toll-like/IL-1 receptor superfamily to innate and adaptive immunity to fungal pathogens in vivo. J. Immunol. 172:3059-3069.

17. Villamon, E., et al. 2004. Toll-like receptor-2 is essential in murine defenses against Candida albicans infections. Microbes Infect. 6:1-7.

18. Yamamoto, Y., Klein, T.W., and Friedman, H. 1997. Involvement of mannose receptor in cytokine interleukin-1beta (IL-1beta), IL-6, and granulocyte-macrophage colony-stimulating factor responses, but not in chemokine macrophage inflammatory protein 1 beta (MIP-1beta), MIP-2, and KC responses, caused by attachment of Candida albicans to macrophages. Infect. Immun. 65:1077-1082.

19. Cambi, A., et al. 2003. The C-type lectin DC-SIGN
(CD209) is an antigen-uptake receptor for Candida albicans on dendritic cells. Eur. J. Immunol. 33:532-538.

20. Klis, F.M., de Groot, P., and Hellingwerf, K. 2001. Molecular organization of the cell wall of Candida albicans. Med. Mycol. 39(Suppl. 1):1-8.

21. Vecchiarelli, A., Puliti, M., Torosantucci, A., Cassone, A., and Bistoni, F. 1991. In vitro production of tumor necrosis factor by murine splenic macrophages stimulated with mannoprotein constituents of Candida albicans cell wall. Cell Immunol. 134:65-76.

22. Tada, H., et al. 2002. Saccharomyces cerevisiae- and Candida albicans-derived mannan induced production of tumor necrosis factor alpha by human monocytes in a CD14- and Toll-like receptor 4-dependent manner. Microbiol. Immunol. 2002:503-512.

23. Jouault, T., et al. 2003. Candida albicans phospholipomannan is sensed through toll-like receptors. J. Infect. Dis. 188:165-172.

24. Gantner, B.N., Simmons, R.M., Canavera, S.J., Akira, S., and Underhill, D.M. 2003. Collaborative induction of inflammatory responses by dectin-1 and Toll-like receptor 2. J. Exp. Med. 197:1107-1117.

25. Brown, G.D., et al. 2003. Dectin-1 mediates the biological effects of beta-glucans. J. Exp. Med. 197:1119-1124.

26. Bates, S., et al. 2005. Candida albicans Pmr1p, a secretory pathway P-type Ca2+/Mn2+-ATPase, is required for glycosylation and virulence. J. Biol. Chem. 280:23408-23415.

27. Munro, C.A., et al. 2005. Mnt1p and Mnt2p of Candida albicans are partially redundant alpha-1,2mannosyltransferases that participate in O-linked mannosylation and are required for adhesion and virulence. J. Biol. Chem. 280:1051-1060.

28. Hobson, R.P., et al. 2004. Loss of cell wall mannosylphosphate in Candida albicans does not influence macrophage recognition. J. Biol. Chem. 279:39628-39635. 
29. Bates, S., et al. 2006. Outer chain N-glycans are required for cell wall integrity and virulence of Candida albicans. J. Biol. Chem. 281:90-98.

30. Singleton, D.R., Masuoka, J., and Hazen, K.C. 2005. Surface hydrophobicity changes of two Candida albicans serotype B mnn 4 delta mutants. Eukaryotic Cell. 4:639-648.

31. Gantner, B.N., Simmons, R.M., and Underhill, D.M. 2005. Dectin-1 mediates macrophage recognition of Candida albicans yeasts but not filaments. EMBO J. 24:1277-1286.

32. Jouault, T., Bernigaud, A., Lepage, G., Trinel, P.A., and Poulain, D. 1994. The Candida albicans phospholipomannan induces in vitro production of tumour necrosis factor-alpha from human and murine macrophages. Immunology. 83:268-273.

33. Jouault, T., et al. 1995. $\beta$-1,2-linked oligomannosides from Candida albicans act as signals for tumor necrosis factor alpha production. Infect. Immun. 63:2378-2381.

34. Torosantucci, A., Chiani, P., and Cassone, A. 2000. Differential chemokine response of human monocytes to yeast and fungal forms of Candida albicans and its relation to the beta-1,6 glucan of the fungal cell wall. J. Leukoc. Biol. 68:923-932.

35. Poulain, D., and Jouault, T. 2004. Candida albicans cell wall glycans, host receptors and responses: elements for a decisive cross-talk. Curr. Opin. Microbiol. 7:342-349

36. Gow, N.A., Brown, A.J., and Odds, F.C. 2002. Fungal morphogenesis and host invasion. Curr. Opin. Microbiol. 5:366-371.
37. Serbina, V., et al. 2003. Sequential MyD88-independent and -dependent activation of innate immune responses to intracellularbacterial infection. Immunity. 19:891-898.

38. Cutler, J.E. 2001. N-glycosylation of yeast, with emphasis on Candida albicans. Med. Mycol. 39S:75-86.

39. Newman, S.L., and Holly, A. 2001. Candida albicans is phagocytosed, killed, and processed for antigen presentation by human dendritic cells. Infect. Immun. 69:6813-6822.

40. Porcaro, I., Vidal, M., Jouvert, S., Stahl, P.D., and Giaimis, J. 2003. Mannose receptor contribution to Candida albicans phagocytosis by murine E-clone J774 macrophages. J. Leukoc. Biol. 74:206-215.

41. Underhill, D.M., and Ozinsky, A. 2002. Toll-like receptors: key mediators of microbe detection. Curr. Opin. Immunol. 14:103-110.

42. Brown, G.D., et al. 2002. Dectin-1 is a major betaglucan receptor on macrophages. J. Exp. Med. 196:407-412

43. Rogers, N.C., et al. 2005. Syk-dependent cytokine induction by Dectin-1 reveals a novel pattern recognition pathway for $\mathrm{C}$ type lectins. Immunity. 22:507-517.

44. Fonzi, W.A., and Irwin, M.Y. 1993. Isogenic strain construction and gene mapping in Candida albicans. Genetics. 134:717-728.

45. Brand, A., MacCallum, D.M., Brown, A.P.J., Gow, N.A.R., and Odds, F.C. 2004. Ectopic expression of URA3 can influence the virulence phenotypes and proteome of Candida albicans but can be overcome by targeted re-integration of URA3 at the RPS10 locus. Eukaryot. Cell. 3:900-909.

46. Kogan, G., Pavliak, V., and Masler, L. 1988. Structural studies of mannans from the cell walls of the pathogenic yeasts Candida albicans serotypes $\mathrm{A}$ and $\mathrm{B}$ and Candida parapsilosis. Carbohydr. Res. 172:243-253.

47. Mueller, A., et al. 2000. The influence of glucan polymer structure and solution conformation on binding to (1-->3)-beta-D-glucan receptors in a human monocyte-like cell line. Glycobiology. 10:339-346.

48. Lowman, D.W., Ferguson, D.A., and Williams, D.L. 2003. Structural characterization of (1-->3)beta-D-glucans isolated from blastospore and hyphal forms of Candida albicans. Carbohydr. Res. 338:1491-1496.

49. Endres, S., Ghorbani, R., Lonnemann, G., Van der Meer, J.W.M., and Dinarello, C.A. 1988. Measurement of immunoreactive interleukin-1 beta from human mononuclear cells: optimization of recovery, intrasubject consistency, and comparison with interleukin-1 alpha and tumor necrosis factor. Clin. Immunol. Immunopathol. 49:424-438.

50. Drenth, J.P.H., et al. 1995. Endurance run increases circulating IL-6 and IL-1ra but downregulates ex vivo TNF- $\alpha$ and IL-1 $\beta$ production. J. Appl. Physiol. 79:1497-1503.

51. Netea, M.G., et al. 1996. Low-density-lipoprotein receptor deficient mice are protected against lethal endotoxinemia and severe Gram-negative infections. J. Clin. Invest. 97:1366-1372. 